\title{
Calcul à la rupture appliqué à la coupe de géomatériaux
}

N. CHALLAMEL

CGES-ENSMP,

35, rue Saint-Honoré, 77300 Fontainebleau

Cet article a pour objet l'étude du chargement maximal d'un géomatériau sollicité par un outil de coupe.

L'analyse est menée en utilisant le formalisme du calcul à la rupture dans le cadre des déformations planes. Le critère de Coulomb est choisi comme critère de rupture du matériau et l'interface est supposée régie par le critère de frottement sec. L'action du poincon orienté rigide idéalisant l'outil peut être réduite à trois paramètres de chargement. L'utilisation du calcul à la rupture permet d'encadrer la frontière des chargements extrêmes dans l'espace des chargements. Nous caractérisons exactement cette frontière en donnant la solution complète dans une certaine plage de paramètres et montrons alors que cette frontière est identique à celle pour laquelle le critère est tronqué en traction. En dehors, nous définissons seulement une région l'encadrant.

Mots clés : calcul à la rupture, coupe de géomatériaux, chargement à trois paramètres, critère de Coulomb, interface de Coulomb, solution complète.

\section{Yield design applied to geomaterial cutting}

This paper sets out to determine the maximal loading of a geomaterial subjected to the action of a cutting tool. The analysis is conducted within the framework of the yield design theory. where plane strain conditions are adopted. The Coulomb criterion is chosen to describe the failure of the material and the interface is assumed to be governed by the dry friction criterion. The tool is modelled as an oriented rigid wedge and its action can be reduced to a loading defined with three parameters. The use of yield design allows to enclose the boundary of the extreme loading. We characterize precisely this boundary. giving the complete solution for a certain range of loading parameters and therefore show, that this boundary is identical to the one for which the criterion is truncated in tension. Outside this range of parameters, we only define an enclosing region in the loading space.

Key words : yield design, geomaterial cutting, three parameters loading, Coulomb criterion. Coulomb interface, complete solution. 


\section{Introduction}

Cette présentation porte sur l'étude de stabilité ou d'instabilité d'une saignée creusée parallèlement à $(\mathrm{Oz})$, de hauteur $h$, chargée par un poinçon incliné frottant. L'objet de cette présentation est de définir des conditions de rupture du matériau chargé comme tel. Les applications d'une telle étude concernent la coupe dans son contexte le plus large, que ce soit la taille des roches (application au forage ou à l'abattage mécanique en général) ou le formage des métaux. Nous orienterons toutefois le travail vers les géomatériaux afin de cibler plus précisément la terminologie. En forage pétrolier par exemple, cette étude permet de quantifier la sollicitation subie par chaque élément de coupe d'un outil de type PDC (Polycristalline Diamond Compact) pour une cinématique donnée. L'intécration de ces actions élémentaires évalue ainsi la résultante au niveau de l'outil, chargement déterminant pour la réponse de la structure foreuse.

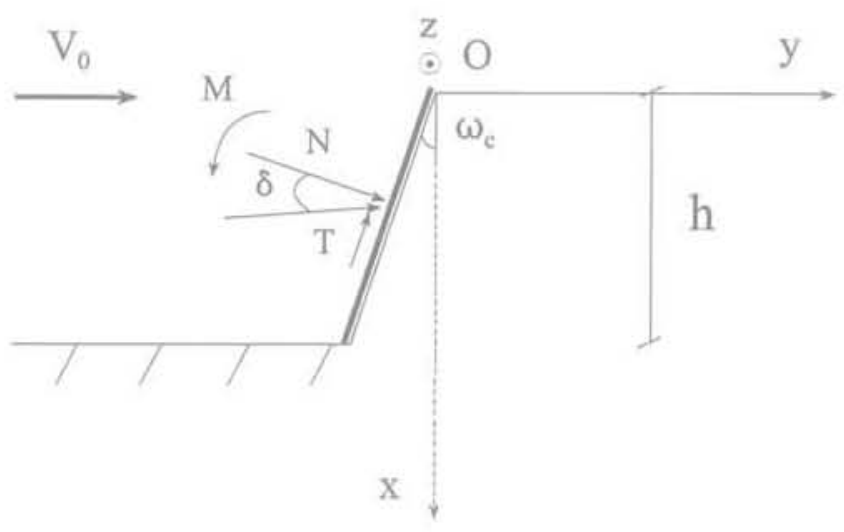

FiG.1 Définition du problème.

Statement of the problem.

Le matériau constitutif est homogène et isotrope. La largeur de la saignée selon $(\mathrm{Oz})$ est supposée grande devant h afin de se restreindre à un calcul en déformations planes. L'analyse est menée dans un cadre quasi statistique pour une configuration géométrique invariante ou suffisamment faible pour ne pas changer la nature du chargement. Le poids propre étant négligé, le chargement du système est constitué uniquement par l'interaction taillant-roche (Fig. 1). Le système est donc régi par des conditions cinématiques au niveau du front de coupe, modélisées par un poinçon orienté, d'angle de coupe $\omega_{c}$, supposé infiniment rigide. Le poinçon se déplace en translation parallèle à (Oy) à la vitesse $V_{0}$. La résultante de l'action du taillant est réduite de manière unique au centre de la saignée aboutissant ainsi à un chargement à trois paramètres (N, T, M), éléments de réduction du torseur des efforts appliqués par le poinçon sur le massif.

En application directe de la théorie du calcul à la rupture, l'analyse de stabilité de la saignée consiste à déterminer en fonction des paramètres du système, le domaine $\mathrm{K}$ de chargement potentiellement supportable par la structure rocheuse dans l'espace (N, T, M) à partir des critères de rupture du matériau.

\section{2}

\section{Formalisme du calcul à la rupture}

Le critère linéaire de Coulomb est choisi comme critère de rupture, décrivant assez fidèlement la rupture de certains géomatériaux. Ce critère utilise deux paramètres : la cohésion C et l'angle de frottement interne $\phi$. Fonction des seules contraintes principales pour un matériau isotrope, il s'écrit dans ce formalisme en tout point :

$$
\begin{gathered}
\mathrm{f}(\underline{\underline{\sigma}})=\sigma_{1}(1+\sin \phi)-\sigma_{3}(1-\sin \phi)-2 \operatorname{Cos} \phi \leq 0 \\
\text { avec } \sigma_{3} \leq \sigma_{2} \leq \sigma_{1}
\end{gathered}
$$

où $\sigma \leq 0$ correspond ici à une compression. Le nombre de traits sous chaque variable symbolise l'ordre du tenseur de la grandeur considérée. Nous supposerons, en outre, que la contrainte principale intermédiaire est la contrainte orthogonale au plan de déformation, ne l'introduisant pas ainsi explicitement dans le critère de rupture.

L'interface entre le taillant et la roche est régie par le critère de frottement sec de Coulomb d'angle $\psi$. On désigne par $\sigma$ la contrainte normale et $\tau$, la contrainte de cisaillement sur la facette de contact taillant-roche. Le critère d'interface s'écrit :

$$
g(\tau, \sigma)=|\tau|+\sigma \tan \psi \leq 0
$$

En reprenant l'étude tribologique de Wojtanowicz (1995) appliquée à l'interface entre un taillant de forage et la roche, $\psi$, peu sensible aux roches testées est en général borné supérieurement par $10^{\circ}$ et est donc bien inférieur aux angles de frottement interne de maintes roches. Ce coefficient tend de plus à s'abaisser avec la vitesse et avec la lubrification (engendrée pour le forage par la boue). Le critère d'interface est alors uniquement conditionné par la loi de frottement sec de Coulomb.

Explicitons le principe fondamental du calcul à la rupture en définissant tout d'abord la terminologie. Le champ de contrainte $\underline{\sigma}$ est dit compatible avec les critères de rupture s'il vérifie (1) en tout point du volume et (2) à l'interface. Un champ est dit Statistiquement Admissible (SA) s'il vérifie les conditions aux limites sur les contraintes et les équations de l'équilibre.

Tous les raisonnements que nous trouverons par la suite sont issus de la proposition logique :

$\exists \underline{\underline{\sigma}}$ SA équilibrant le chargement (N, T, M) et compatible avec les critères de rupture $\Leftrightarrow(N, T, M) \in K$.

Deux types d'approches sont envisageables pour encadrer la frontière de K, surface des chargements extrêmes. L'approche de cette frontière qui définit la limite de stabilité potentielle du système dans la géométrie fixée peut se construire par l'intérieur ou par l'extérieur, qui encadre respectivement le domaine par défaut et par excès. Les deux méthodes sont donc nécessairement complémentaires, et l'utilisation exclusive d'une des approches est difficilement exploitable.

Historiquement, les premiers résultats concernant l'encadrement de la frontière de $\mathrm{K}$ pour ce mode de chargement sont obtenus dans les années 40 par Merchant (1944), en application à la coupe des métaux. Ces travaux en liaison avec l'effort industriel conséquent 
des États-Unis durant la Deuxième Guerre mondiale, s'inspirent de la méthode du prisme de Coulomb, écrite à la fin du XVIII' siècle, équivalente à une approche cinématique par bloc rigide. Merchant définit ainsi une première borne extérieure (la frontière surfacique de $\mathrm{K}$ dans $(\mathrm{N}, \mathrm{T}, \mathrm{M})$ est réduite à un point pour $\mathrm{M}=0$ et en supposant une hypothèse de glissement à l'interface taillant roche). L'encadrement de la frontière générale pour $\mathrm{M}=0$ (comprenant en particulier celui du point obtenu par Merchant) est toutefois amélioré dans les années 70 par Nguyen Minh (1975), utilisant alors la méthode cinématique des caractéristiques, en s'inspirant du problème du poinçonnement (voir par exemple Sokolovsky, 1965 ou Pariseau and Fairhurst, 1967). Les travaux de Lebrun (1978), puis de Sellami s'attachèrent à élargir la méthode du prisme de Coulomb à une configuration tridimensionnelle. Bien que l'hypothèse des déformations planes puisse être discutée dans certaines configurations de coupe, l'encadrement de la frontière de K en déformations planes est un problème encore largement ouvert jusqu'alors. C'est donc ce cadre de réflexion que nous choisissons dans cette étude. Nous montrons que nous pouvons compléter la frontière cinématique par les caractéristiques par une approche statique, dans certains cas et donnons une formulation générale de la surface de chargement potentiellement supportable ou d'un pavé l'encadrant.

Nous définirons d'abord le domaine de chargement potentiellement supportable pour $\mathrm{M}=0$ et l'étendrons au cas $\mathrm{M} \neq 0$ par la méthode de la largeur de poinçon réduite (voir par exemple Salençon et Pecker, 1995). Nous nous intéressons dans un premier temps à l'encadrement par défaut de la frontière de chargement potentiellement supportable, appelée aussi approche statique.

\section{3.}

\section{Approche statique}

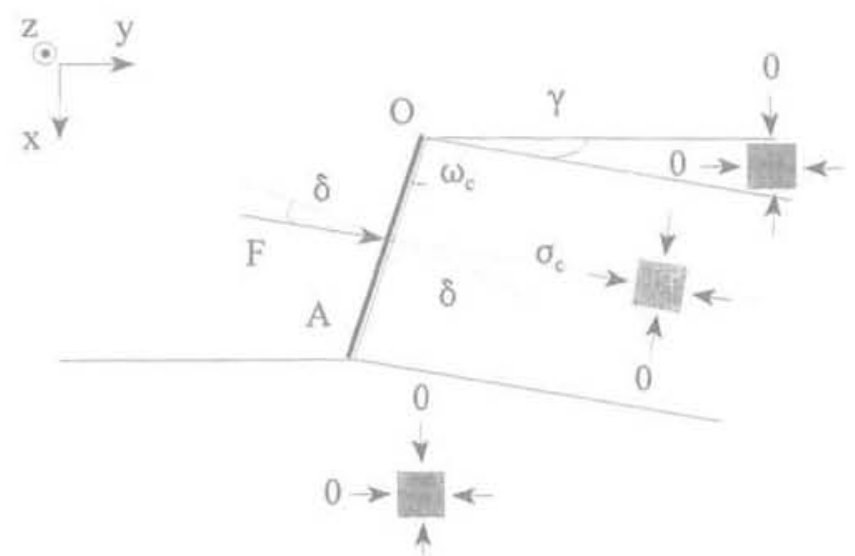

FIG. 2 Solution champ constant par morceaux. Static solution with a discontinuous stress field.

L'objet est d'exhiber un champ de contrainte qui équilibre le chargement du poinçon, champ qui soit SA et qui soit compatible avec les critères de rupture. La méthode générale consiste à diviser le volume de la structure continue en sous-domaines élémentaires sur lesquels on définit le champ de contrainte désiré. Ces champs vont être construits analytiquement de manière simple, constants par morceaux, ou continus (par la méthode des caractéristiques).

\section{1}

\section{Solution champ constant par morceaux}

Les champs de contraintes sont illustrés ici par leurs axes principaux. Le volume total est partitionné en trois sous-domaines dont deux en contact avec les surfaces libres pour lesquels le champ de contrainte est identiquement nul (Fig. 2). Le dernier domaine est caractérisé par une bande de champ uniforme. Ce champ atteint le critère de rupture et sa contrainte principale majeure est nulle. $\sigma_{c}$ égal à $-\frac{2 \mathrm{C} \cos \Phi}{1-\sin \Phi}$, désigne ici la résistance à la compression simple. Par construction, ce champ pourra répondre aux conditions limites sur la facette de contact OA par détermination de l'orientation de cette bande. Un tel champ est solution du problème si $\gamma=\omega_{c}-\delta \geq 0$. Pour $\delta \geq 0$, cette construction n'est valable que pour une certaine plage de valeurs de l'angle de coupe. La recherche d'une frontière pour $\delta \in[-\psi, \psi]$ avec ce champ impose $\omega_{c}-\psi \geq 0$. Autrement dit, si cette dernière condition n'est pas remplie, la construction ne sera valable que sur une portion du domaine que nous cherchons à couvrir. Le champ de contrainte construit dans tout le volume, constant par morceaux, est ainsi statistiquement admissible et compatible avec les critères de rupture.

$$
\begin{aligned}
& N(\delta)=h \frac{2 C \cos \phi}{1-\sin \phi} \frac{\cos \delta}{\cos \omega_{c}} \cos \delta \\
& T(\delta)=h \frac{2 C \cos \phi}{1-\sin \phi} \frac{\cos \delta}{\cos \omega_{c}} \sin \delta \\
& M=0
\end{aligned}
$$

Le domaine défini par la frontière décrite par les équations ci-dessous, est alors intérieur à $\mathrm{K}$. Cet encadrement de la frontière réelle peut être nettement amélioré pour $\delta \geq 0$ en utilisant la méthode des caractéristiques qui permet de faire varier continument la direction des contraintes principales pour un champ qui atteint le critère en tout point.

\section{2}

\section{Méthode des caractéristiques}

On partitionne de même le domaine total en sousdomaines élémentaires (Fig. 3). Pour un de ces domaines (cône de base OE), le champ de contraintes est partiellement construit à l'aide de la méthode des caractéristiques. En écrivant les équations de l'équilibre et le critère de rupture atteint en tout point de la zone considérée, nous aboutissons à la résolution d'un système hyperbolique quasi linéaire aux dérivées partielles. Le long de lignes caractéristiques appelées classiquement $\alpha$ et $\beta$, le système se réduit à une différentielle ordinaire. La démonstration dans notre configuration s'inspire largement du problème de la butée d'une paroi lisse sur un massif plastique pour le critère de Tresca (Salençon, 1972), puis étendu au critère de Coulomb (Salençon, 1973). Des techniques de prolongement du champ sont décrites par Bishop (1953) ou Shield (1954) pour le critère de Tresca. 
La construction dans tout le domaine s'effectue en deux temps (Fig. 3), D'abord, le champ de Prandtl de base OE et d'ouverture $\Omega$ est prolongé à l'aide de la méthode de Shield, dont la démonstration est donnée pour le matériau de Coulomb par Salençon (1973) pour un domaine de variation d'angle de frottement interne qui englobe largement le domaine concerné par la plupart des roches $\left(\phi \leq 76^{\circ}\right)$. Ceci nous définit donc un secteur angulaire d'ouverture $2 \Omega$ (et $\Omega \leq \omega$ ) à la frontière duquel le champ de contrainte est identiquement nul. Le champ de contrainte dans le triangle OAE, d'angle au sommet $\omega_{-}-\Omega$, est uniforme et continu à la frontière OE. Il est alors possible de compléter ce champ à partir de la frontière AE, par une bande de champ constant uniforme, discontinu à la frontière $\mathrm{AE}$, de contrainte principale majeure nulle. Un tel champ est compatible avec le critère de Coulomb si $\sigma_{1} \geq \sigma_{e}$, soit la condition :

$$
\mathrm{e}^{2 \sin \phi} \leq \frac{1+\sin \phi}{1-\sin \phi}
$$

Cette condition est en général remplie et est d'autant moins pénalisante que l'angle de frottement interne est faible. En effet, pour $\phi=60^{\circ}$, l'inégalité se traduit par $\Omega \leq 43^{\circ}$. Les angles de coupe $\omega_{\text {c }}$ étant rarement supérieurs à $30^{\circ}$ (pour le forage par exemple) et

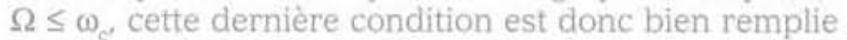
dans la majorité des cas. N'eût-ce point êté le cas, il eût toujours été possible d'employer de même un champ de Prandtl prolongé par la méthode de Shield par exemple pour le cône de base AE. La condition limite sur la facette $O A$, donnée par l'angle $\delta$, amène à une équation transcendante en $\Omega$, qui détermine alors de manière unique le champ de Prandtl :

$$
\begin{aligned}
& \sin \phi \sin \left(2 \omega_{c}-2 \Omega\right) e^{2 \Omega \tan \phi}- \\
& \tan \delta\left[\mathrm{e}^{2 \Omega \tan \phi}\left(1+\sin \phi \cos \left(2 \omega_{\mathrm{c}}-2 \Omega\right)\right)-(1-\sin \phi)\right]=0
\end{aligned}
$$

On en déduit ainsi les paramètres $(\mathrm{N}(\delta), \mathrm{T}(\delta))$ potentiellement supportables par la structure rocheuse et un nouvel encadrement par défaut de $\mathrm{K}$ pour $\mathrm{M}=0$.

$$
\mid \begin{aligned}
& N(\delta)=\frac{h}{\cos \omega_{c}} \frac{\cos \phi}{1-\sin \phi} e^{20 \tan \phi} \frac{\sin \left(2 \omega_{c}-2 \Omega\right)}{\tan \delta} \\
& T(\delta)=\frac{h}{\cos \omega_{c}} \frac{C \cos \phi}{1-\sin \phi} e^{20 \tan \varphi} \sin \left(2 \omega_{c}-2 \Omega\right) \\
& M=0
\end{aligned}
$$

La recherche d'une frontière pour $\delta \in[-\Psi, \Psi]$ avec ce champ impose de même $\omega_{-}-\Psi \geq 0$.

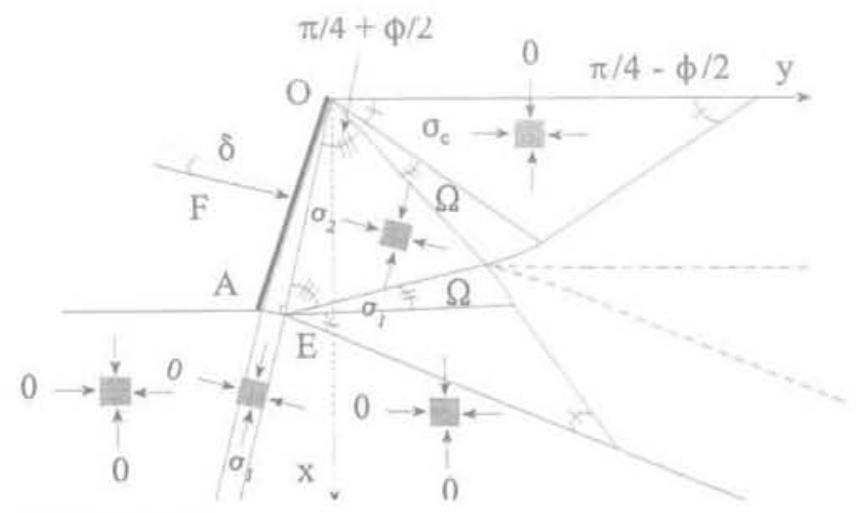

FG. 3 Approche statique par les caractéristiques.

Static approach using the characteristics method.
Le cas limite $\delta=0$ correspondant à la solution classique du poinçon lisse est donné par : $\mathrm{N}(0)=$

$$
\frac{h}{\cos \omega_{c}} \frac{\operatorname{Cos} \phi}{\sin \phi(1-\sin \phi)}\left[(1+\sin \phi) e^{2 \omega_{c} \tan \phi}-1+\sin \phi\right]
$$

Après avoir élaboré des champs de contrainte SA et compatibles avec les critères de rupture pour encadrer par l'intérieur la frontière de rupture dans l'espace $(\mathrm{N}$, T), nous nous penchons sur son encadrement par l'extérieur ou approche cinématique.

\section{4}

\section{Approche cinématique}

\section{1}

\section{Présentation générale}

La méthode s'appuie sur l'écriture du principe des puissances virtuelles pour un chamip virtuel cinématiquement admissible. La présentation générale de la méthode est donnée par Halphen et Salençon (1987). Nous la particularisons à notre configuration, afin de mieux cibler la démarche. Pour que l'instabilité du massif soit certaine, il faut qu'on ait incompatibilité entre l'équilibre statique sous le chargement (N, T, M) (ou F et $\delta$ pour $\mathrm{M}=0$ ) et les capacités de résistance du matériau. La frontière du domaine $\mathrm{K}$ est alors approchée par l'extérieur en écrivant cette incompatibilité.

Le champ virtuel de vitesse $\underline{V}$ cinématiquement admissible est choisi identiquement nul pour la zone complémentaire à $\Omega_{0}$ (Fig. 4). Pour ce dernier volume, il est défini par un champ de vitesse de déformation au sein même de l'élément et par un champ de discontinuité de vitesse $\mathrm{V}_{\mathrm{u}}$ à l'interface taillant roche et le long de la coupure fictive $\Sigma$.

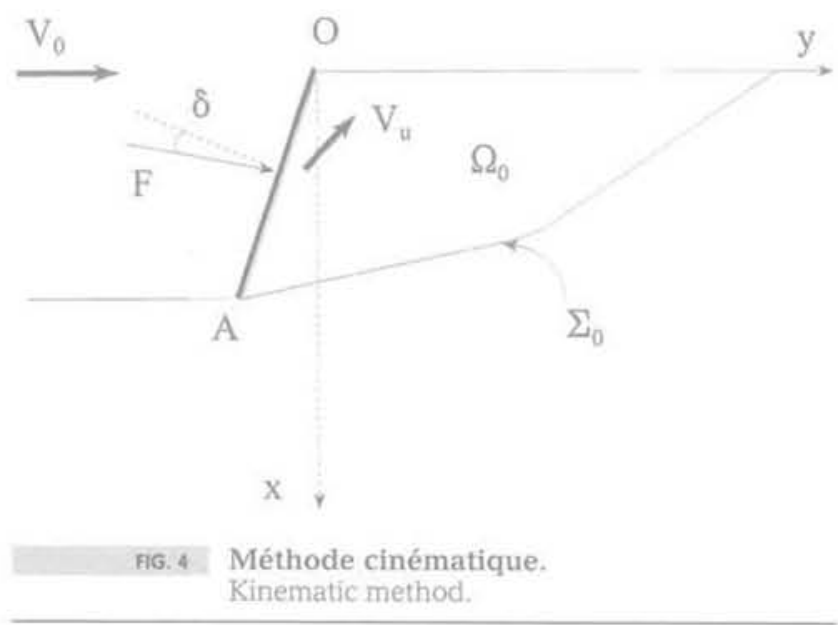

Le principe des travaux virtuels s'écrit pour un charnp de contrainte statistiquement admissible et pour le champ virtuel cinématiquement admissible $\underline{V}$ dans le repère fixe $(\mathrm{Oxyz})$ :

$$
\begin{aligned}
& \int_{\Omega_{0}} \underline{\sigma}(\underline{x}) \cdot \underline{d}(\underline{x}) d \Omega_{0}+\int_{\Sigma_{0}}[[\underline{V}(\underline{x})]] \cdot \underline{\underline{\sigma}}(\underline{x}) \cdot \underline{n}(\underline{x}) d S \\
& \quad+\int_{O A}[[\underline{V}(\underline{x})]] \cdot \underline{\sigma}(\underline{x}) \cdot \underline{n}(\underline{x}) d S=\underline{F} \cdot\left(\underline{V_{0}}+\underline{V_{u}}\right)
\end{aligned}
$$


où : $\mathrm{n}(\mathrm{x})$ vecteur normal à la surface considérée. $\mathrm{d}(\mathrm{x})$ champ de vitesse de déformations,

[[V(x)]] discontinuité du champ de vitesse à la traversée de OA ou $\Sigma_{0}$.

Introduisons maintenant le concept de puissance résistante maximale, à partir des critères de rupture du géomatériau et de l'interface, respectivement $\mathrm{f}$ et $\mathrm{g}$.

$$
\begin{aligned}
& \pi_{\mathrm{f}}(\underline{\underline{\mathrm{d}}})=\operatorname{Sup}(\underline{\underline{\sigma}} \underline{\underline{\mathrm{d}}} ; \mathrm{f}(\underline{\underline{\sigma}}) \leq 0) \\
& \pi_{1}(\underline{\underline{n}},[\underline{\underline{V}]]})=\operatorname{Sup}([\underline{\mathrm{V}}]] \cdot \underline{\underline{\sigma}} \cdot \underline{\mathrm{n}} ; \mathrm{f}(\underline{\underline{\sigma}}) \leq 0) \\
& \pi_{g}(\underline{n},[[\underline{V}]])=\operatorname{Sup}([\underline{\underline{V}}] \cdot \underline{\underline{\sigma}} \cdot \underline{\mathrm{n}} ; \mathrm{g}(\underline{\underline{\sigma}} \cdot \underline{\mathrm{n}}) \leq 0)
\end{aligned}
$$

Posons maintenant

$$
\mathrm{P}(\underline{\mathrm{V}})=\int_{\Omega} \pi_{f}(\underline{\mathrm{d}}) \mathrm{d} \Omega_{0}+\int_{\Sigma} \pi_{f}(\underline{\mathrm{n}},[[\underline{\mathrm{V}}]]) \mathrm{d} S+\int_{\mathrm{OA}} \pi_{0}(\underline{\mathrm{n}},[[\underline{\mathrm{V}}]]) \mathrm{dS}
$$

Nous pouvons ainsi écrire : compatibilité entre l'équilibre quasi statique sous $\mathrm{F}$ et les capacités de résistance du matériau $\Rightarrow \underline{F} \cdot\left(\underline{V_{0}}+\underline{V_{u}}\right) \leq P(\underline{V})$.

La contraposée de cette proposition logique nous amène à : $\underline{\mathrm{F}} \cdot\left(\underline{\mathrm{V}_{\mathrm{O}}}+\underline{\mathrm{V}_{\mathrm{u}}}\right)>\mathrm{P}(\underline{\mathrm{V}}) \Rightarrow$ l'instabilité du massif rocheux est certaine.

Le premier terme correspond à la puissance extérieure; le second terme à la borne supérieure de la puissance dissipée pour le champ cinématique admissible choisi. C'est ce deuxième terme que nous allons identifier maintenant, pour différents champs cinématiques virtuels.

L'expression explicite des puissances maximales pour $\mathrm{f}$ et $\mathrm{g}$, en fonction des paramètres des critères, peut être trouvée dans Halphen et Salençon (1987) et est appelée ci-dessous. $V_{n}$ et $V_{\text {}}$ désignent les composantes respectivement normales et tangentielles de [IV]] par rapport à la facette définissant I'interface.

$$
\begin{aligned}
& \mid \pi_{f}(\underline{\underline{d}})=+\infty \sin \underline{\underline{d}}<<\left(\left|\mathrm{d}_{1}\right|+\left|\mathrm{d}_{2}\right|\right) \\
& \pi_{\mathrm{f}}(\underline{\underline{d}})=\mathrm{C} \cdot \operatorname{cotan} \Phi \text { trd } \sin o n \\
& \left.\mid \pi_{f}(\underline{n},[\underline{\underline{V}}] \mathrm{I})=+\infty \text { si }[[\underline{\mathrm{V}}]] \cdot \underline{\mathrm{n}}<\mid[\underline{\mathrm{V}}]\right] \mid \sin \Phi \\
& \pi_{r}(\underline{n},[[\underline{V}]])=C \cdot \operatorname{cotan} \phi[[\underline{V}]] \cdot \underline{n} \sin o n \\
& \left\{\begin{array}{l}
\pi_{0}(\underline{n},[[\underline{V}]])=+\infty \text { si } V_{n}<V_{1} \tan \psi \\
\pi_{0}(\underline{n},[[\underline{V}] \bar{I})=0 \text { sinon }
\end{array}\right.
\end{aligned}
$$

\section{2}

\section{Méthode cinématique par les caractéristiques}

Nous reprenons le raisonnement présenté par Nguyen Minh en 1975, exposant la méthode cinématique par les caractéristiques. Toutefois, nous n'introduisons par la rhéologie en tant que telle dans le raisonnement, et restons ainsi dans le formalisme strict du calcul à la rupture. L'objet est d'exhiber un mode de déformation dans tout le volume satisfaisant les conditions aux limites sur les vitesses et permettant de borner les fonctions $\pi$. Le champ de vitesse de déformations dans $\Omega_{0}$ va maintenant être construit (Fig. 5). Nous renvoyons le lecteur à Nguyen Minh (1974) pour l'élaboration plus détaillée d'une telle construction et montrons simplement qu'il répond aux exigences requises.
Les notations données dans l'approche statique pour $\alpha$ et $\beta$ sont reprises.

Posons $\left(V_{\alpha^{\prime}}, V_{\beta}\right)$, les valeurs algébriques des projections orthogonales des vitesses sur les axes tangents à $\alpha$ et $\beta$.

Ce champ vérifie les équations différentielles (appelées équation de Geiringer pour le matériau de Tresca):

$$
\begin{aligned}
& d V_{\alpha}-\left(V_{\alpha} \tan \phi+\frac{V_{\beta}}{\cos \phi}\right) d \theta=0 \text { le long de } \alpha \\
& d V_{\alpha}+\left(\frac{V_{\alpha}}{\cos \phi}+V_{\beta} \tan \phi\right) d \theta=0 \text { le long de } \beta
\end{aligned}
$$

où $\theta$ est défini par $\theta=\left(O x, e_{\alpha}\right), e_{\alpha}$ et $e_{\beta}$ étant les vecteurs unitaires tangents aux lignes $\alpha$ èt $\beta$ en un point d'intersection des lignes. On a en outre $\left(e_{n}, e_{3}\right)=\pi / 2+\phi$.

Les lignes $\beta$ étant rectilignes dans la zone OABCD (qui correspond au domaine $\Omega_{0}$ ), $V_{\beta}$ est constante le long de chacune de ces lignes (et confinue à la frontière $\mathrm{OBCD}$ rigide) et est donc nulle dans toute la zone plastique.

$V_{0}$ est constante le long d'une ligne $\alpha$, rectiligne et est supposée constante sur OA; ainsi dans tout le triangle $\mathrm{OAB}, \mathrm{V}_{\alpha}$ est égale à $\mathrm{V}_{\mathrm{\alpha} 0}$. Les valeurs de $\mathrm{V}_{\alpha}$ dans le reste de la zone déformée s obtiennent en exprimant la continuité de $V_{\alpha}$ en $A B$ et $A C$ et en respectant les relations pour les vitesses issues de la relation différentielle. Dans l'éventail de Prandtl, d'ouverture $\Omega, V_{\text {, est }}$ constante le long d'un rayon vecteur et égale à $V_{\alpha 0}^{\alpha} e^{\text {utano }}$ où $\mu$ angle de rayon vecteur $\mu \in[0, \Omega]$. Pour le champ $\mathrm{OCD}, \mathrm{V}_{\alpha}$ est égale à $\mathrm{V}_{\alpha 0} \mathrm{e}^{\mathrm{m} \text { ran } \Phi}$.

Un tel champ est cinématiquement admissible et annule I'inégalité (8) dans tout le volume. Le calcul de la puissance $\mathrm{P}(\mathrm{V})$, lorsqu'elle est finie, amène à la mème frontière que dans l'approche statique par les caractéristiques pour $\delta \geq 0$ mais cette fois approchée par l'extérieur. Le raisonnement est toutefois valable quel que soit le signe de $\delta$ et donne une frontère cinématique sur tout l'espace recherché.

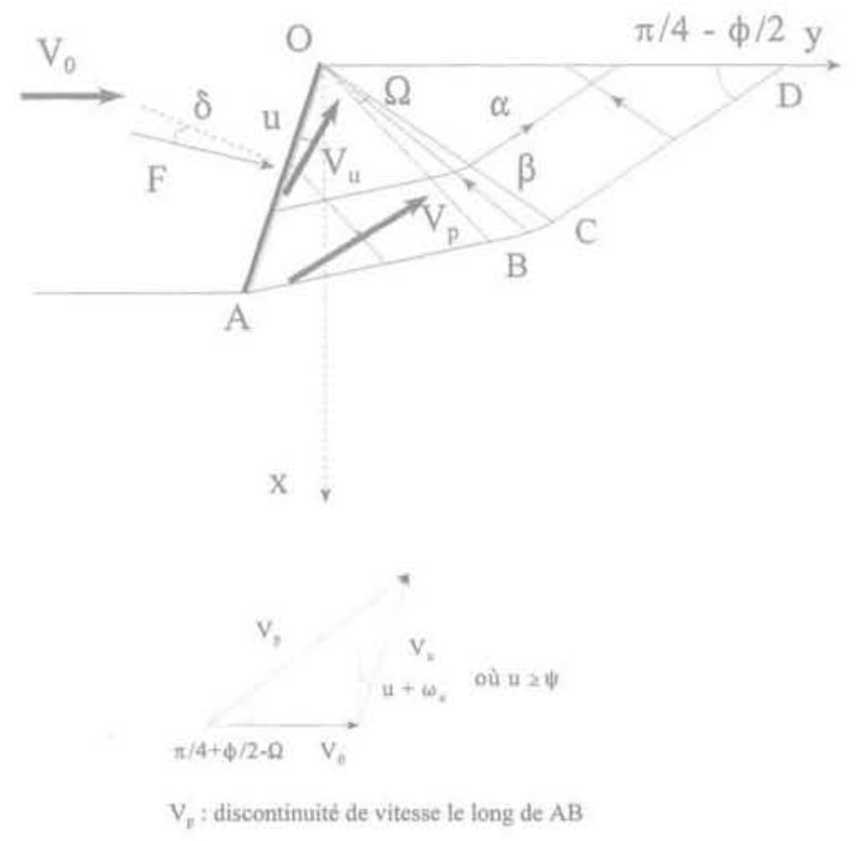

FG.5 Champ cinématique par les caractéristiques.

Construction of the kinematic field using the characteristics method. 


\section{Système avec critère tronqué en traction}

La solution est dite complète pour $\delta \geq 0$ (les deux encadrements sont confondus) et nous avons ainsi identifié exactement une portion de la frontière de K. Le raisonnement effectué précédemment nous permet de surcroît d'obtenir une information sur la charge de ruine du même système mais avec un critère de rupture tronqué en traction. En effet, pour un tel système l'approche par l'intérieur est identique car seule l'information en compression a été utilisée pour la construction du champ de contrainte désiré. Or le domaine $\mathrm{K}$ du système tronqué en traction est inclus dans celui du système non tronqué que nous avons traité précédemment (par inclusion du critère de rupture tronqué dans le critère non tronqué). La frontière de $\mathrm{K}$ avec critère de Coulomb tronqué en traction est donc la même pour $\delta \geq 0$ que celle identifiée pour le critère sans troncature. La caractérisation du matériau à la traction, en général peu aisée, n'est donc pas un enjeu majeur (le problème est bien conditionné) pour cette gamme de paramètres de chargement. Ainsi pour les roches, dont on sait que la courbe intrinsèque a tendance à s'incurver dans le domaine de la traction, la frontière définie reste valable dans cette configuration de chargement.

\section{4}

\section{Méthode cinématique par bloc rigide}

Bien que moins intéressante pour l'encadrement de $\mathrm{K}$, il nous a paru important de rappeler l'expression de la frontière obtenue par la méthode cinématique par bloc rigide, ne serait-ce que pour la situer par rapport à celle par les caractéristiques. Nous reprenons le raisonnement fondamental explicité en 4.1. en choisissant un champ cinématique par bloc rigide et une frontière de glissement rectiligne. En optimisant le paramètre définissant l'orientation de cette ligne, la méthode, simple d'usage, donne les paramètres de chargement non potentiellement supportables pour $\phi-\delta+\omega_{c}<\frac{\pi}{2}$

$$
\mid \begin{aligned}
& N(\delta)=h \frac{2 C \cos \phi}{1-\sin \left(\phi-\delta+\omega_{c}\right)} \cos \delta \\
& T(\delta)=h \frac{2 C \cos \phi}{1-\sin \left(\phi-\delta+\omega_{c}\right)} \sin \delta \\
& M=0
\end{aligned}
$$

\section{5}

\section{Représentation graphique}

\section{1}

\section{Cas $M=0$}

Nous représentons graphiquement les frontières définies dans les chapitres précédents. L'utilisation de la propriété de convexité du domaine de chargement potentiellement supportable qui découle elle-même de la propriété de convexité du critère de rupture, permet de réduire l'incertitude sur la frontière de ce domaine pour $\delta<0$, à partir des deux frontières statiques par les caractéristiques et par champ constant par morceaux.
L'approche statique par champ constant par morceaux sert donc uniquement de construction à la frontière statique rectiligne pour $\delta<0$. L'approche cinématique par bloc rigide est d'autant moins intéressante par rapport à l'approche cinématique par les caractéristiques que $\delta$ est petit. Notons que pour $\omega_{c}=\psi$, l'écart entre les deux bornes cinématiques s'annule.

Dans la représentation, on a supposé une différence faible entre $\omega_{c}$ et $\Psi$, amenant à un écart à peine perceptible entre les deux bornes cinématiques pour $\delta=\Psi$. De même, on suppose implicitement $\omega_{\mathrm{f}} \geq \Psi$ (Fig. 6). Pour $\omega_{c}<\Psi$, les frontières statique et cinématique par les caractéristiques sont tronquées pour $\delta \in\left[\omega_{c} \Psi\right]$ et une autre frontière peut être obtenue par l'approche cinématique par bloc rigide.

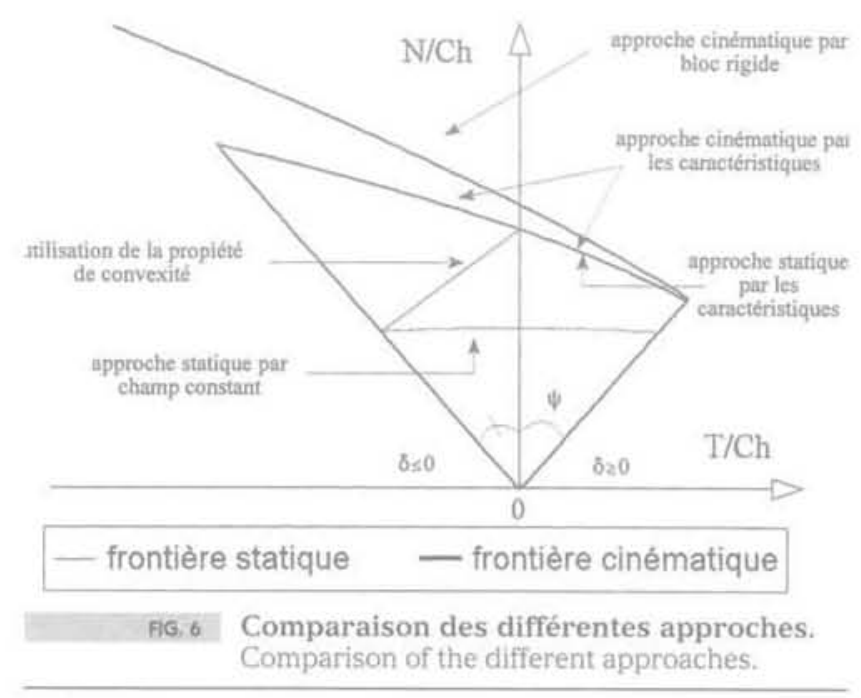

La figure 7 est extraite de la figure 6 plus générale, le résultat est synthétisé pour $\mathrm{M}=0$.

Il pourrait sans doute être judicieux, afin de diminuer les incertitudes sur les frontières, notamment pour $\delta<0$, de construire des champs de contrainte désirés de manière numérique, par exemple par la méthode des éléments finis (Pastor, 1978).

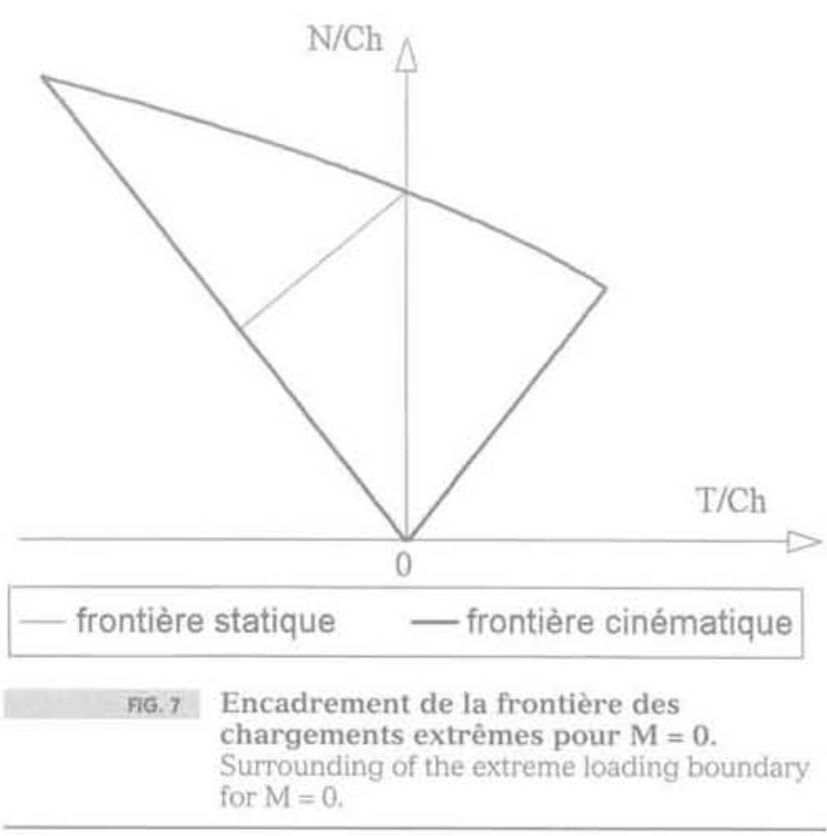




\section{Cas $M \neq 0$}

En raison des conditions cinématiques imposées par la physique du problème, l'approche cinématique par les caractéristiques reste invariante quel que soit le moment $\mathrm{M}$. Le poinçon n'ayant qu'un mouvement de translation, sa rotation virtuelle est nulle dans l'écriture du principe des puissances virtuelles (présentation générale de la méthode cinématique du 4.1). La puissance extérieure développée par le moment est donc identiquement nulle, se ramenant au calcul pour $\mathrm{M}=0$.

\subsection{5}

\section{Méthode statique de la largeur de poinçon réduite}

Posons $M=$ Ne où $|e| \leq 1 / 2$ avec $l=h / \cos \omega$ et e est l'excentricité, grandeur orientée. En utilisant la méthode de la largeur de poinçon réduite qui consiste à utiliser les mêmes champs de contrainte précédemment définis sur une portion de la surface de contact entre le taillant et le géomatériau, la frontière statique à moment constant se déduit de celle à moment nul par homothétie de rapport $1-2 \mid$ e $\mid / 1$ et de centre O (la représentation graphique suppose de même $\left.\omega_{c} \geq \Psi\right)$.

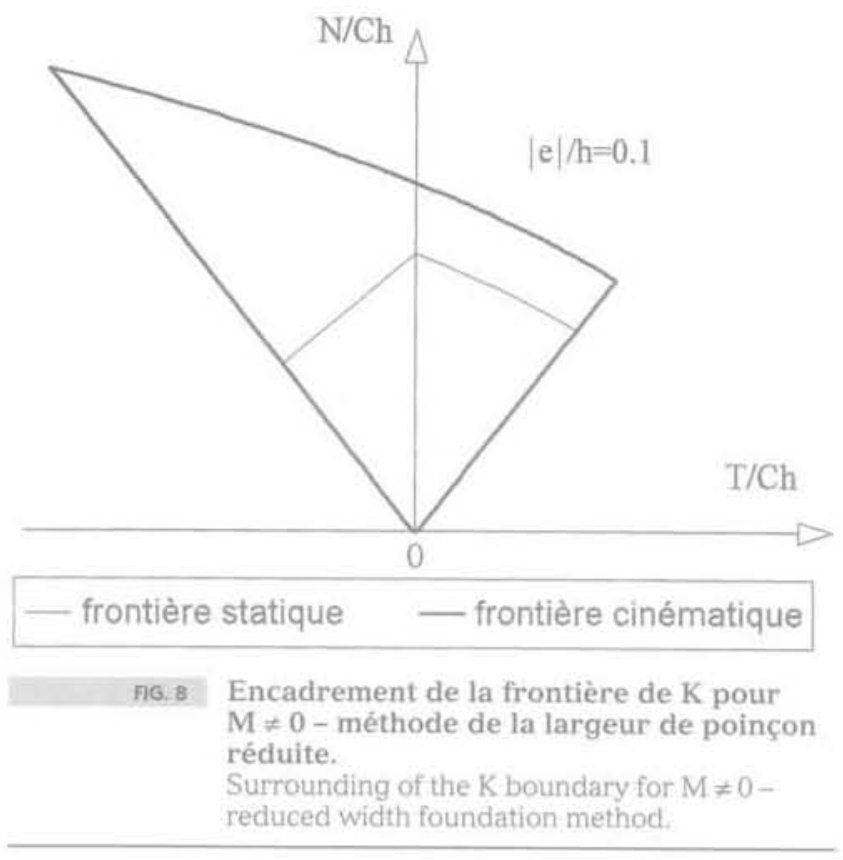

L'écart entre les deux frontières statique et cinématique est ainsi d'autant plus important que e est grand (Fig. 8).

\section{2}

\section{Méthode statique par champ mixte}

Cette méthode améliore la frontière statique construite par la méthode de la largeur de poinçon réduite pour $\mathrm{M} \leq 0$ et $\delta \leq 0$ (de manière moins marquée pour $M \geq 0$ et $\delta \geq 0$ ). La méthode est décrite pour cette première plage de paramètres et le raisonnement est similaire dans la deuxième plage. Une telle famille de champs est définie par trois paramètres : $\Omega$, $\gamma$ et $h_{0}$ (Fig. 9). $\Omega$, le paramètre d'ouverture du champ de Prandtl, est borné par $\omega$. La zone 0 est caractéristique du champ de Prandtl déjà introduit, pour lequel la contrainte tangentielle le long de la face d'attaque est nulle. Nous retrouvons le champ défini dans l'approche statique par champ constant pour la zone 1.

Les valeurs de contraintes normales et tangentielles le long de la face d'attaque s'écrivent alors:

$$
\mid \begin{aligned}
& \sigma_{0}=\frac{-C \cos \phi}{\sin \phi(1-\sin \phi)}\left((1+\sin \phi) e^{2 \sin \phi}-1+\sin \phi\right) \\
& \sigma_{1}=-\frac{2 C \cos \phi}{1-\sin \phi} \frac{1+\cos 2 \gamma}{2} \\
& \tau_{1}=-\frac{2 C \cos \phi}{1-\sin \phi} \frac{\sin 2 \gamma}{2}
\end{aligned}
$$

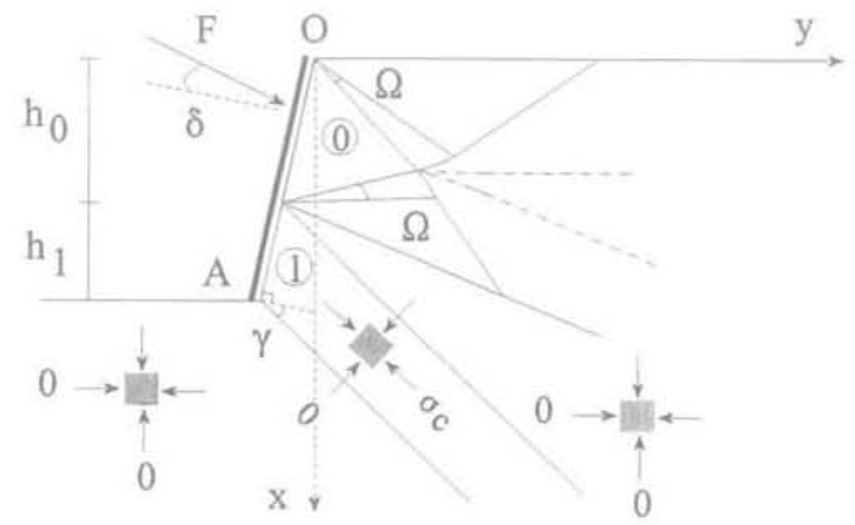

HG.9 Approche statique mixte $-\mathrm{M} \leq 0-\delta \leq 0$. Mixed static approach $-\mathrm{M} \leq 0-\delta \leq 0$.

Les deux conditions limites en $\delta$ (définissant l'orientation du chargement) et en e (définissant le moment), fonction des contraintes paramétrées des zones 0 et 1 sur la face d'attaque, se traduisent par :

$$
\begin{aligned}
& \frac{h_{0}}{h}=\frac{\sigma_{1} \tan \delta+\tau_{1}}{\sigma_{1} \tan \delta+\tau_{1}-\sigma_{0} \tan \delta} \\
& \frac{e}{h}=\frac{h_{0}}{h} \frac{h_{1}}{h} \frac{\left(\sigma_{1}-\sigma_{1}\right)}{2\left(\sigma_{0} \frac{h_{0}}{h}+\sigma_{1} \frac{h_{1}}{h}\right)} \frac{1}{\cos \omega_{c}}
\end{aligned}
$$

Pour chaque valeur de $\delta$, la formulation (13) donnant $\mathrm{e}$, aboutit à une équation transcendante en $(\gamma, \Omega)$ résolue numériquement. Pour de faibles valeurs de e, le champ optimal qui vérifie les conditions aux limites est atteint pour $\gamma=\Omega$, se ramenant à un problème uniquement conditionné. Au-delà, la valeur maximale $\Omega=\omega$ est optimale et l'équation (13) se résout alors en $\gamma$ dans l'intervalle $\left[\omega_{c^{\prime}}, \pi / 2\right]$. La figure 10 représente l'analogue de la figure 8 mais avec l'amélioration ci-dessus définie. Quel que soit e négatif, cette frontière est toujours plus intéressante que celle obtenue par l'approche de la largeur de poinçon réduite, d'autant plus d'ailleurs que $\delta$ est petit. Le cas $M \geq 0$ et $\delta \geq 0$ s'obtient à partir du cas précédent en inversant les zones 0 et 1 et en reprenant la méthodologie pour les faibles valeurs de e $(\gamma$ borné par $\left.\omega_{c}\right)$. 


\section{2 .3}

\section{Visualisation dans le plan ( $N$, M)}

Les figures 11 et 12 représentent l'encadrement de $\mathrm{K}$ dans le plan (N, M) à $\delta$ constant. Pour $\delta$ négatif arbitrairement choisi à $-\Psi$, nous retrouvons l'accroissement du domaine pour $\mathrm{M} \leq 0$, permettant notamment de nous prononcer de manière plus précise sur le paramètre N potentiellement supportable. L'utilisation de la propriété de convexité de (N, T, M), conservée lorsque

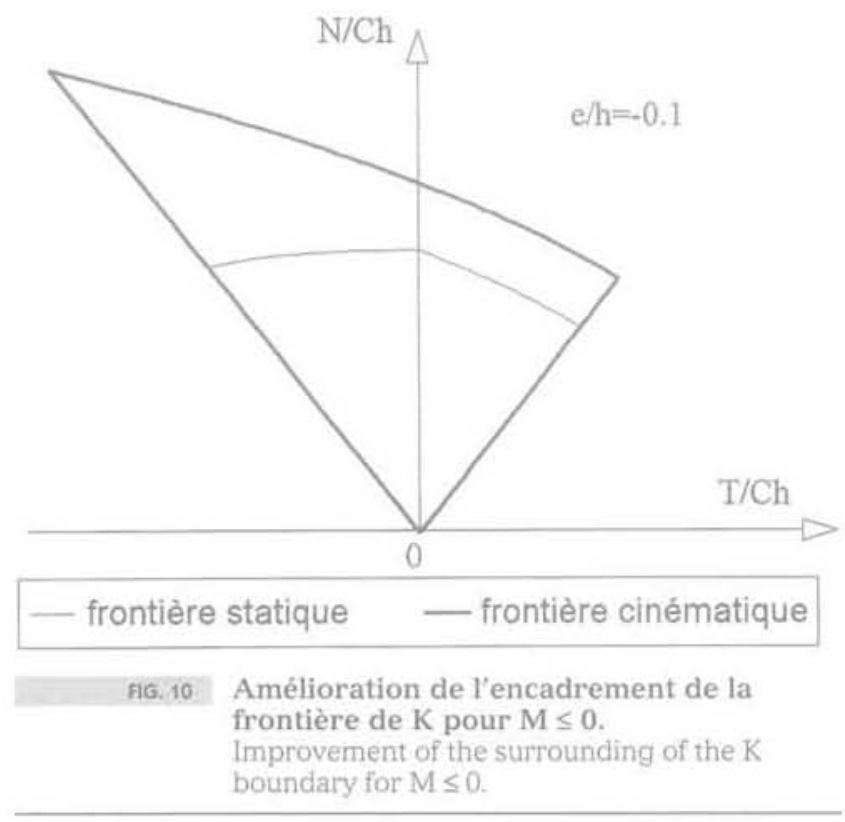

l'on coupe le domaine $\mathrm{K}$ par les plans $\mathrm{M}=\mathrm{Ne}$ ou $\mathrm{N}=\mathrm{T} \tan \delta$, permet de surcroit d'améliorer la frontière recherchée pour $\mathrm{M}=0$, de manière peu significative il est vrai.

Du fait que la contrainte le long de la face d'attaque est nécessairement une compression, l'excentricité est bornée par l'intervalle $[-1 / 2,+1 / 2]$. Cette condition se traduit par l'inégalité : $|\mathrm{M}| \leq \mathrm{N}^{*} 1 / 2$. Ainsi, une frontière cinématique est décrite dans le plan de variables adimensionnelles (N/Ch, $\mathrm{M} / \mathrm{Ch}^{2}$ ) par les deux droites passant par l'origine et de coefficient $\pm 1 /\left(2 \mathrm{CO}_{\mathrm{c}}\right)$.

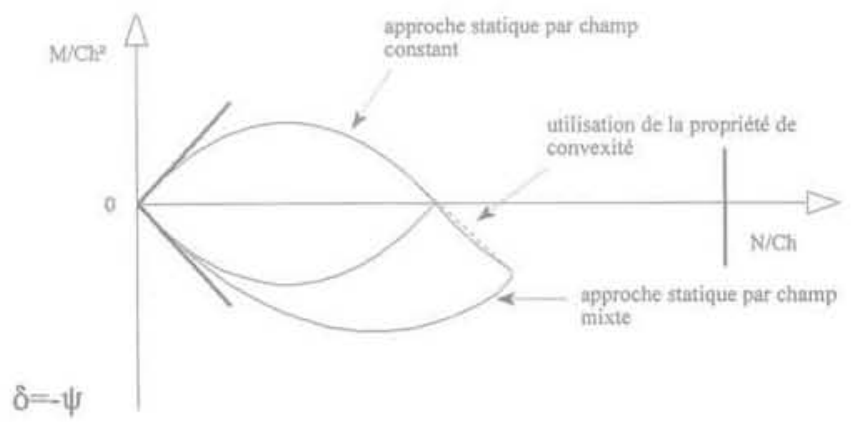

frontiere statique _frontière cinématique

FiG, 11 Encadrement de la frontière de $\mathrm{K}$ pour $\delta \leq 0$.

Surrounding of the $\mathrm{K}$ boundary for $\delta \leq 0$.
Une autre frontière cinématique (Fig. 12), qui passe par le point extrême donné par la solution complète, est parallèle à l'axe des ordonnées. En effet, à $\delta$ constant, la frontière cinématique par les caractéristiques est invariante avec $\mathrm{M}$.

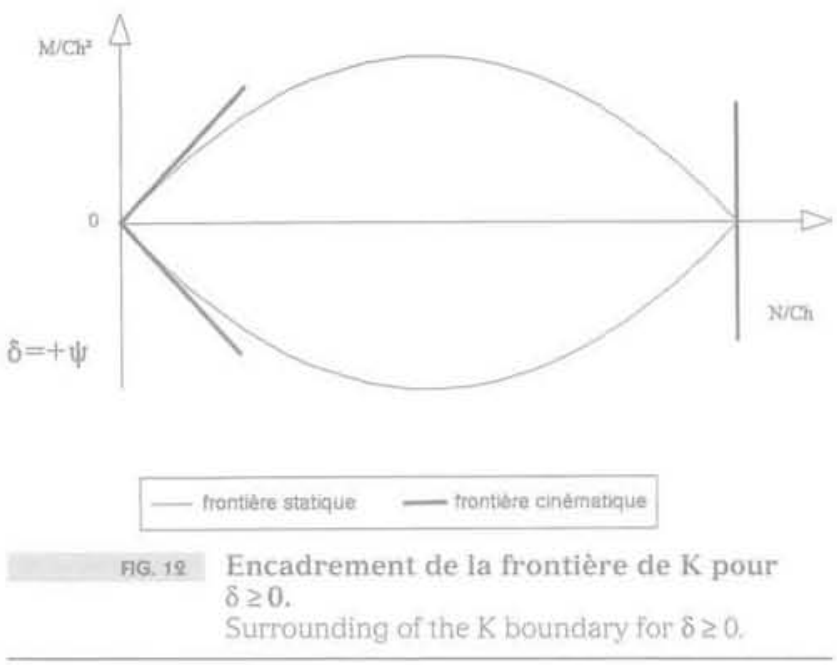

5.3

\section{Interprétation dans le cadre de l'analyse limite}

Jusque-là, et c'est la puissance de l'approche, nous avons établi la frontière de ruine (connue exactement ou encadrée), uniquement à partir de considérations portant sur un critère de rupture en contrainte sans faire d'hypothèse sur la loi de comportement de la roche. Autrement dit, sans s'occuper de la rhéologie, la frontière précédemment définie donnera un majorant de la frontière de ruine effective de la structure. Lobjectif en coupe étant de détruire la matière, la majoration obtenue va dans le même sens que le dessein recherché.

Le critère de rupture peut aussi être assimilable à un critère d'écoulement plastique (bien que le critère de Coulomb soit rarement associé à ce type de comportement sauf pour les matériaux pulvérulents comme le sable) et l'interprétation du précédent calcul en supposant un comportement du géomatériau élastoplastique parfait appartient au domaine de l'analyse limite. On montre que sỉ le matériau étudié admet une loi d'écoulement de type standard (le potentiel plastique est confondu avec la fonction seuil de plasticité), on a unicité du chargement limite (Mandel, 1966). Deux conséquences importantes de ce théorème concernent l'indépendance đu chargement limite (chargement de ruine du système élastoplastique associé) aux caractéristiques élastiques du matériau d'une part, et l'indépendance de ce chargement limite aux contraintes initiales. De plus, la solution complète apporte l'information supplémentaire suivante: le théorème d'unicité du champ de contrainte, qui découle du théorème d'association, dans le plan de déformation, est applicable dans la zone déformée, moyennant certaines conditions sur la contrainte intermédiaire (Mandel, 1965 pour le matériau de Tresca et le raisonnement est identique pour celui de Cou- 
lomb). Ceci n'entraîne évidemment pas que la solution complète est unique dans tout le volume car il est possible de compléter le champ en général de plusieurs manières.

En réalité, les roches ou sols modélisés par une surface de charge de type Coulomb ont rarement une loi d'écoulement standard (Maier, 1979; Radenkovic, 1961) ainsi que leurs interfaces. Le théorème d'unicité n'est alors plus valable. La frontière de rupture déterminée précédemment donne ainsi une majoration de la frontière réelle de ruine élastoplastique, qui dépend alors du trajet de chargement (Radenkovic. 1961). De plus, les roches à de faibles niveaux de confinement présentent souvent une fragilité qui impose la prudence quant à l'interprétation de cette frontière. Le comportement plus ductile de ces dernières sous hautes pressions de confinement justifie toutefois l'utilisation du formalisme du calcul à la rupture.

\section{6}

\section{Conclusion}

Le calcul à la rupture nous a permis de définir des frontières des chargements potentiellement admis- sibles d'un matériau de Coulomb sollicité par un poinçon orienté frottant. Cette frontière est exactement connue dans une certaine plage de ces paramètres, ayant alors pu confondre la frontière statique et cinématique. En dehors de cette configuration, l'encadrement se traduit par un domaine dans l'espace tridimensionnel (N, M, T). La visualisation de ce domaine est donnée graphiquement par projection de la région recherchée sur les plans d'excentricité constante ou d'orientation de chargement constant, définissant des aires d'incertitude en général d'autant plus réduites que le moment est faible. Toutefois, pour $T \leq 0$ et $M \leq 0$, nous avons pu resserrer l'encadrement, en construisant un champ de contrainte par une méthode mixte (par les caractéristiques et par une bande uniforme). Les frontières cinématiques sont importantes lorsque l'on cherche à détruire le géomatériau, comme c'est le cas dans la coupe, car elle donne un majorant de la sollicitation réelle, allant dans le même sens que l'objectif désiré. De plus et bien que ne bénéficiant d'aucune justification mathématique, le champ virtuel ayant permis de construire la borne cinématique est souvent associé avec le mécanisme réel observé, permettant au besoin de faire évoluer cette borne et de décrire le processus effectif de coupe.

\section{HEMERCIIMENTS}

Nous remercions vivement $M$. Salençon pour les conseils qu'il nous a prodigués, en particulier pour la construction des approches statiques.

\section{Bibliographie}

Bishop J.F,W, - " On the complete solution to problems of deformation of a plasticrigid material i. Journal of the Mechanics and Physics of Solids, vol. 2, 1953. p. 43-53.

Halphen B., Salençon J. - Elastoplasticité, Paris, Presse des Ponts, 1987

Lebrun M. - "Etude théorique et expérimentale de l'abattage mécanique; application à la conception de machines d'abattage et de creusement $n$. Thèse de docteur-ingênieur. ENSMP, 1978.

Maier G, Hueckel I. - « Non associated and coupled flow rules of elastoplasticity for rock-Lilke materials 3 . Int. J. Rock. Min. Sci. \& Geomech., abstr. vol. 16, 1979, p. 77-92

Mandel J. - a Sur l'unicité du champ des contraintes lors de l'équilibre limite dans un milieu rigide plastique D. C.R.Ac.Sc., Paris, 261, 1965, p. 35-37.

Mandel J. - Mécanique des millieux continus, Paris, Gauthier-Villars, 1966.
Merchant M.E. - $\alpha$ Mechanics of metal cutting process II - Plasticity conditions in orthogonal cutting, 3 J. Appl. Phys., $16(6), 1945$, p. 318-324.

Nguyen Minh D. - a Contribution à l'étude de la taille des roches $x$. Revue de IInstitut français du pétrole, vol. XXX, n²3. 1975, p. $473-498$.

Pariseau W.G., Fairhsut C. - 4 The forcepenetration characteristic for wedge penetration into rock 1). Int. J, Rock. Min. Sci. \& Geomech., vol. 4. 1967, p. 165-180.

Pastor J, - " Analyse limite : détermination numérique de solutions statiques complètes : application du talus vertical $\mathrm{p}$. Journal de mécanique appliquée, vol. 2 , $n^{\circ}$ 2. 1978 .

Radenkovic $\mathrm{D},-\alpha$ Theorèmes limites pour un matériau de Coulomb à dilatation non standardisée ». C.R.Ac.Sc., Paris, 252, 1961, p. 103-4104.

Salençon J. - « Butée d'une paroi lisse sur un massif plastique: solutions statiques ». Journal de Mécanique vol. $11, n^{\circ} 1,1972, p .135-146$.

Salençon J. - « Prolongement des champs de Prandtl dans le cas du matériau de Coulomb 3. Archives of Mechanics, 25, 4, 1973, p. 643-648.

Salençon J. Pecker A - « Ultimate bearing capacity of shallow foundations under inclined and eccentric loads. Part 1: purely cohesive soil x. European Journal of Mechanics, A/Solids, vol. 14, $\mathrm{n}^{\circ} 3$. 1995. p. 349-375.

Shield R.T. - " Plastic potential theory and Prandtl bearing capacity solution n. J.Appl. Mech. Trans. ASME, 21, 1954, p. 193-194,

Sokolovsky V.V. - Statics of granular media, New York, Pergamon Press, 1965. Wojtanowicz A.K. Kuru E. - \& An experimental study of sliding friction between PDC drill cutters and rocks $\%$. Int. J. Rock. Mech. Min. Sci. \& Geomech. abstr., vol. 32, $\pi^{\circ} 3,1995$, p. 277-283. 